\title{
Structure of V-ATPase from mammalian brain
}

\author{
Y Abbas $^{1}$ \\ ${ }^{1}$ The Hospital for Sick Children, Toronto \\ yazan.abbas@gmail.com
}

Signal propagation across the chemical synapse between the axon terminal of a presynaptic neuron and the dendrite of a postsynaptic neuron requires the regulated release of neurotransmitters from vesicles into the cleft. The synaptic vesicle membrane is energized by the proton pumping activity of vesicular- or vacuolar-type ATPases (V-ATPases) to allow transporters to load vesicles with neurotransmitters. In V-ATPases, ATP hydrolysis in the catalytic V1 region drives rotation of a central rotor subcomplex and leads to proton translocation through the membraneembedded VO region. V-ATPase activity is regulated by reversible separation of the $\mathrm{V} 1$ and $\mathrm{VO} \neg$ regions, with ATP hydrolysis inhibited in the isolated V1 complex and the VO complex becoming impermeable to protons. Fusion of synaptic vesicles with the presynaptic membrane requires separation of the V1 and VO regions but it is not known how these events are coordinated. The mammalian $\mathrm{V} 1$ region contains subunits A3B3CDE3FG3H, and the $\mathrm{VO}$ region is thought to be composed of acxc-de as well as ATP6AP1, also known as Ac45, and ATP6AP2, also known as the (pro)renin receptor. ATP6AP2/PRR is involved in several signalling pathways including the reninangiotensin system for regulating blood pressure and electrolyte balance and Wnt signalling in stem cells and embryo development. The precise arrangement of subunits in the mammalian VO region remains unclear. Further, mammals have multiple isoforms of some subunits in both V1 and VO that are expressed in a tissue-dependent and cellular-compartment-dependent way, complicating their analysis. We isolated rat synaptic vesicle V-ATPase from rat-brain membranes through its interaction with SidK, a Legionella pneumophila effector protein. CryoEM allowed construction of an atomic model, revealing the mammalian V-ATPase architecture and composition, and defining the enzyme's ATP:H+ ratio as 3:10. The c-ring encloses the transmembrane anchors for cleaved ATP6AP1/Ac45 and ATP6AP2/PRR. The structure shows how ATP6AP1/Ac45 and ATP6AP2/PRR enable assembly of the enzyme's catalytic and membrane regions.

Acta Cryst. (2020). A76, a123 